\title{
Lead Protection and Positioning Splints before Brachytherapy in Maxillofacial Prosthodontics: A Case Report
}

\author{
Wijdane EL Hawari ${ }^{1}$, Eddarif Oumaima ${ }^{2}$, Nadia Merzouk ${ }^{3}$, Oussama Bentahar ${ }^{4}$
}

\begin{abstract}
The prevalence of head and neck cancers increases worldwide, the therapeutic arsenal of its management mainly includes surgery, radiotherapy (RTH), and the combination of these two therapies. Chemotherapy is also used as an adjuvant treatment. Brachytherapy (BT) is indicated for localized head and neck cancers of the tumors of the lips and the nasal pyramid, the oral cavity, and the oropharynx. Lead protection and positioning splints are generally used when BT is provided. They help protect uninvolved tissues as the dose that will be delivered is important. They are made before BT and positioned during irradiation. This case report presents a clinical case of a patient with lip cancer for treatment with BT and for which a lead protection splint for maxillary arch protection and mandibular positioning splint was given.

Keywords: Brachytherapy, Lead protection, Positioning splints, Prosthetic rehabilitation, Radio, Removable.

International Journal of Prosthodontics and Restorative Dentistry (2021): 10.5005/jp-journals-10019-1314
\end{abstract}

\section{BACKGROUND}

The prevalence of head and neck cancers increases worldwide, the therapeutic arsenal of its management mainly includes surgery, radiotherapy (RTH), and the combination of these two therapies. Chemotherapy is also used as an adjuvant treatment. ${ }^{1}$ Brachytherapy (BT) and external beam radiation therapy (EBRT) are the two types of radiation techniques that are used clinically. ${ }^{2}$

External beam irradiation (EBI) results in unnecessary radiation exposure of the adjacent normal tissues. ${ }^{3}$ In contrast to BT which delivers a high dose in a short time and a small volume, it reduces the irradiation of surrounding normal tissues. The main indications of BT for localized head and neck cancers are the tumors of the lips and the nasal pyramid, the oral cavity, and the oropharynx. ${ }^{4}$

The uses of the prosthesis including radiation source carriers, perioral cone positioning stents, shielding stents, tissue recontouring stents, tissue bolus compensators, and may limit the complications following head and neck RTH. ${ }^{5}$ These devices are used to adjust the position, or protect the tissues, or help in the effective administration of irradiation to the affected sites. ${ }^{6}$

Lead protection and positioning splints are generally used when BT is provided. They help protect uninvolved tissues as the dose that will be delivered is important. They are made before BT and positioned during irradiation.

Lead protection splints are commonly made from Lipowitz's alloy or Wood's metal (eutectic alloy of $50 \%$ bismuth, $26.7 \%$ lead, $13.3 \%$ tin, and $10 \%$ cadmium) popularly known as Cerrobend. ${ }^{7}$ Positioning splints allow the displacement of normal tissues away from the areas of high-dose irradiation. ${ }^{8}$ A multidisciplinary approach is important for an optimal therapeutic strategy, ${ }^{9}$ indeed, the collaboration between the maxillofacial prosthodontist and the radiotherapist/oncologist is the guarantee of the proper management of these patients. ${ }^{10}$

This case report presents a clinical case of a patient with lip cancer for treatment with BT and for which a lead protection splint for maxillary arch protection and mandibular positioning splint was made.

\begin{abstract}
1,3,4 Department of Prosthodontics, Faculty of Dental Medicine, University Mohammed V, Rabat, Morocco

${ }^{2}$ Department of Radiotherapy, National Institute of Oncology, University Mohammed V, Rabat, Morocco

Corresponding Author: Wijdane EL Hawari, Department of Prosthodontics, Faculty of Dental Medicine, University Mohammed V, Rabat, Morocco, Phone: +212 637642136, e-mail: wijdane.elhawari@ um5.ac.ma

How to cite this article: El Hawari W, Oumaima E, Merzouk N, et al. Lead Protection and Positioning Splints before Brachytherapy in Maxillofacial Prosthodontics: A Case Report. Int J Prosthodont Restor Dent 2021;11(2):105-107.

Source of support: Nil

Conflict of interest: None
\end{abstract}

\section{Case Description}

A 93-year-old man presented to the center of consultations and dental treatments of Rabat for fabrication of lead protective stents for adjuvant BT for carcinoma of the upper lip. The biopsy exeresis and anatomopathological examination revealed a welldifferentiated squamous carcinoma of the upper lip.

Dental assessment, intraoral photography, and mouth-opening measures have been obtained to assess the feasibility of stents. An intraoral examination revealed completely edentulous maxillary and mandibular arches with the favorable alveolar ridge, vestibular sulci, and the palate for the retention of the protective stents.

The objective of prosthetic management is to fabricate maxillary and mandibular stents to ensure an adequate clinical target volume delineation and proper conditions of adaptation of these stents during RTH. The oral positioning stents were customized and ensure position maintenance during RTH sessions. Maxillary stents were made to shield the surrounding structures which are adjacent to radiation. The mandibular stents were fabricated to displace the mandible away on the side for which the stent was placed. 


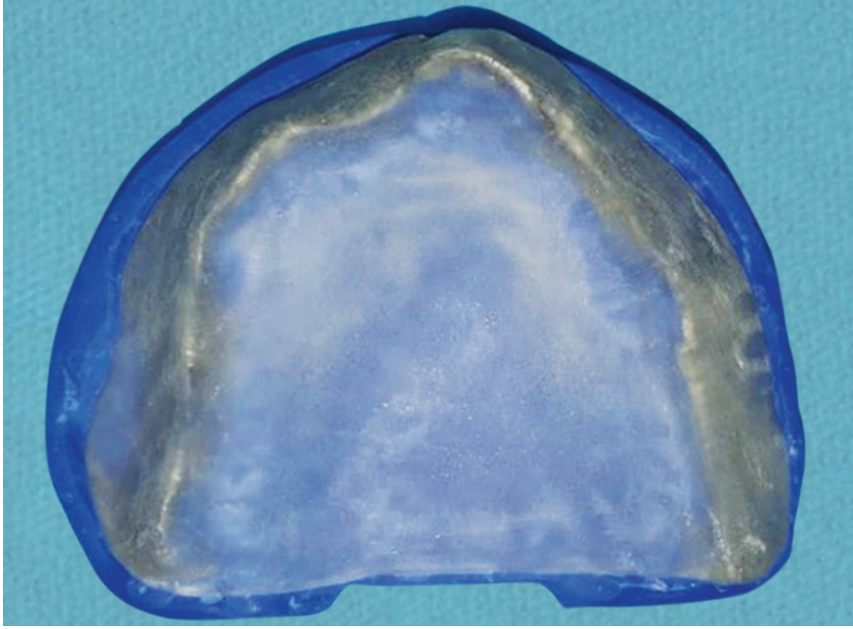

Fig. 1: Lead protection splint on the primary maxillary model

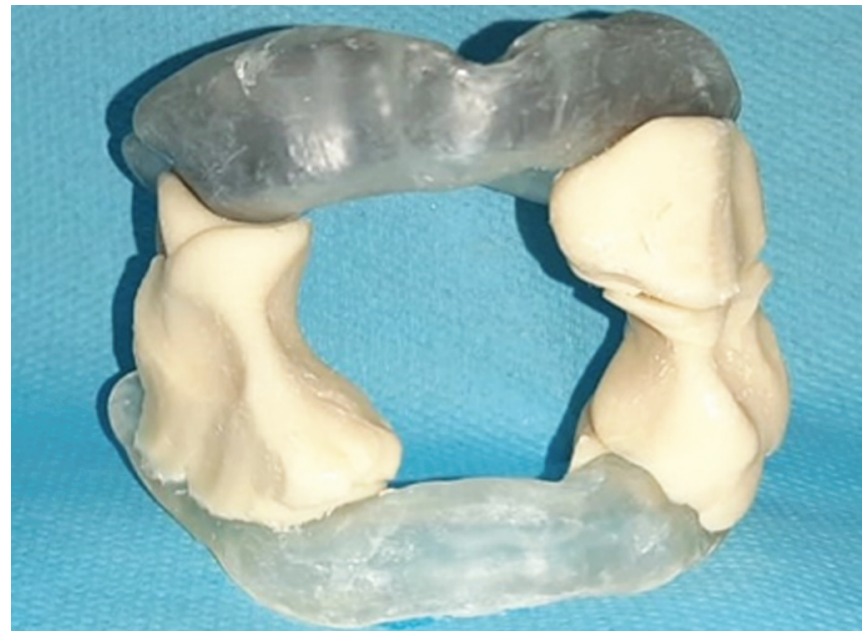

Fig. 3: Maxillary lead stent and mandibular positioning stent

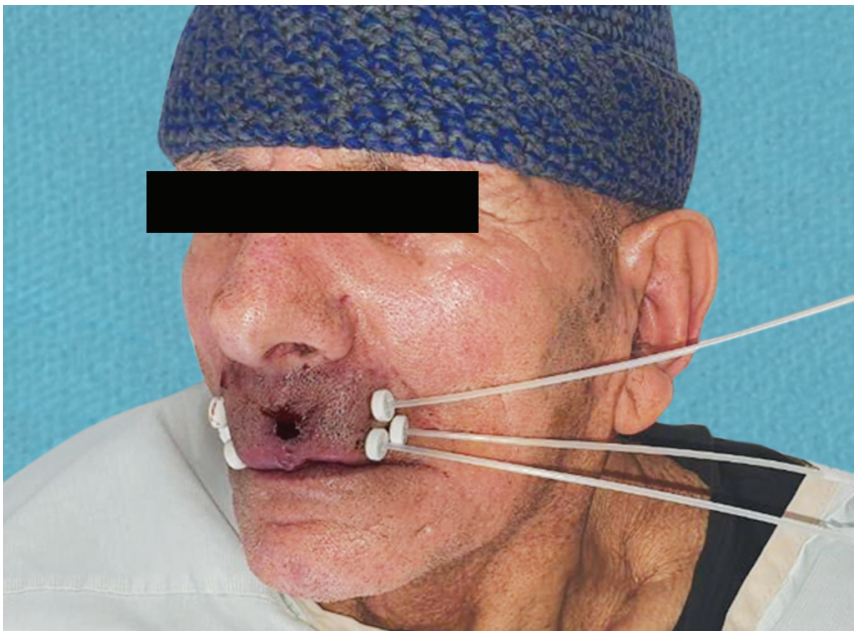

Fig. 5: Needles inserted on the upper lip

The distance between the incisors of the oral positioning stents usually varied from 1 to $3 \mathrm{~cm}$ depending on maximum open mouth, length, and space needed to separate surrounding structures. ${ }^{11}$ This distance was fixed at $2 \mathrm{~cm}$, using Unifast ${ }^{\oplus}$ resin as a wedge.

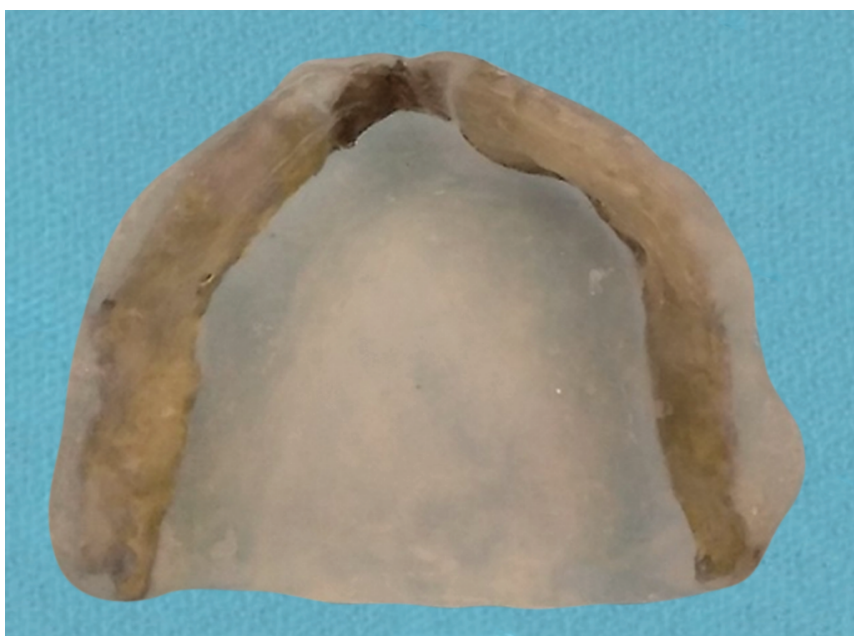

Fig. 2: View of the intrados of lead protection splint

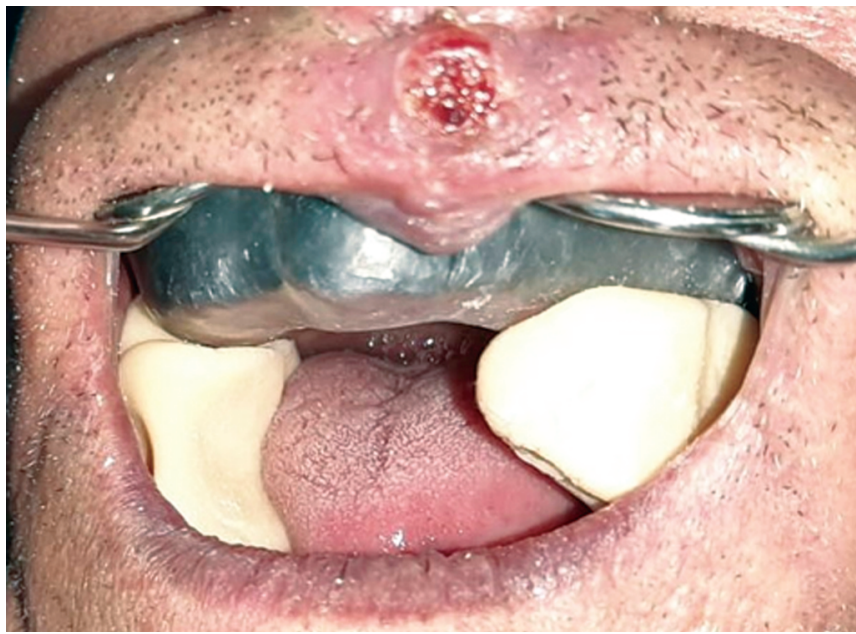

Fig. 4: Intraoral view of the protective stents

Primary models were obtained from the maxillary and mandibular preliminary impressions. On the maxillary model, over a transparent resin plate, a standard lead block at the vestibular level along the maxillary arcade was placed and covered with a second layer of resin (Fig. 1). For the mandibular model, a transparent resin plate was adapted to the entire mandibular support surface (Fig. 2). Careful trimming and polishing were done for both stents. The wedge was sinned in two parts to allow easy removal of the two stents and at the same time realized grooves to facilitate a repetitive repositioning of the mandibular stents (Fig. 3).

The splint was placed in the oral cavity and checked for proper adaptation (Fig. 4). The radiation technique used was BT with a total irradiation dose of $60 \mathrm{~Gy}$ in 5 fractions. The needles were inserted at the upper lip (Fig. 5), and the stents were placed in the oral cavity before each session of irradiation.

\section{Discussion}

There are two different modalities of RTH, external and interstitial. External radiation therapy is used to deliver high doses of radiation to tumors that are located within $6 \mathrm{~cm}$ of the skin surface. Brachytherapy is interstitial RTH that delivers high doses (up to 20,000 rads) of radiation over a short distance for a 
short time period (10-15 hours). ${ }^{12}$ Head and neck RTH treatment results in healthy tissue damage near the tumor site. ${ }^{11}$ The need for oral radiation stents is important for both modalities of irradiation.

The study of Yangchen et al. evaluated the efficacy of Cerrobend shielding stents in minimizing the potential adverse effects of radiation on oral tissues in buccal carcinoma patients and has concluded that Cerrobend alloy can be used effectively to reduce the adverse effect associated with EBRT. ${ }^{13}$

Tongue-depressing stents can provide a patient's position fixed for multiple RTH stents but it may increase the interocclusal height and provide a bulky sensation under wearing. ${ }^{6}$ Grant et al. investigated the potential benefit of tongue deviating oral stent (TDOS) use on dosimetric parameters in unilateral intensitymodulated radiation therapy (IMRT), the study had concluded that the use of TDOS for unilateral treatment of well-lateralized tonsil cancers was associated with oral mucosa sparing for IMRT. ${ }^{14}$

In the present case report, a block of interocclusal resin was used to spare the tongue and the mandible away from the radiation site. As for the materiel of realization of the radiation stents, lead or Cerrobend was used. Jabbari et al. concluded that in terms of the protection of the opposite side of the oral cavity, the uniform lead shield produces better shielding compare to other materials (Plexiglas, acrylic resin, silicone, and plaster). In the present case, a standard block of lead was used to protect the opposite site which was the maxillary arch and soft and hard palate.

Farajollahi et al. investigated the influence of either Cerrobend and lead block methods on central axis dose distribution of photon beam a cobalt unit and concluded that the use of melt shielding methods is a very easy and fast shield-making technique with no differences in percent depth dose, absolute and skin dose between lead and Cerrobend block shielding methods. ${ }^{15}$

Doi et al. studied the utility and stability of intraoral stents during IMRT and concluded that interfractional setup errors in anterior-posterior and 3D dimensions can be significantly reduced by using intraoral stents. ${ }^{16}$ Intraoral scanner methodologies or computed tomography can be used to create these radiation stents, it affects clinical flow, increases patient comfort, and enhances prediction of head and neck RTH dosimetric value. ${ }^{15}$

Zaid et al. established a digital workflow for the custom designing and 3D printing mouth-opening, tongue-depressing stents for patients receiving RTH for head and neck cancer and concluded that the application of this rapid and efficient digital workflow can expend the use of these devices. ${ }^{9}$ A systematic literature review conducted by Chen et al. evaluated the efficacy of positioning stents in preventing oral complications after RTH and concluded that there is a need for more high-quality and prospective trials with long-term follow-up to support it. ${ }^{17}$

The complications of RTH can be decreased or avoided effectively with the help of these stents. It is then wise to prevent complications before it occurs. The collaboration between the radiotherapist and the maxillofacial prosthodontist is necessary and constitutes an essential step if these stents are indicated.

\section{References}

1. El Hawari W, Rokhssi H, Azhari M, et al. Radiotherapy and management in maxillofacial prosthodontic: part 1 - before radiotherapy. Integr J Med Sci 2021. 8. DOI: 10.15342/ijms.2021.386.

2. Skowronek J. Current status of brachytherapy in cancer treatment short overview. JCB 2017;9(6):581-589. DOI: 10.5114/jcb.2017.72607.

3. Takácsi-Nagy Z, Martínez-Mongue R, Mazeron J-J, et al. American Brachytherapy Society task group report: combined external beam irradiation and interstitial brachytherapy for base of tongue tumors and other head and neck sites in the era of new technologies. Brachytherapy 2017;16(1):44-58. DOI: 10.1016/j.brachy.2016.07.005.

4. Peiffert $D$, Coche-Dequéant $B$, Lapeyre $M$, et al. Curiethérapie des cancers de la tête et du cou: synthèse des recommandations européennes et principales indications. Cancer/Radiothérapie 2018;22(4):359-366. DOI: 10.1016/j.canrad.2017.12.005.

5. Aggarwal $H$, Kumar P. Radiation stents: minimizing radiation-induced complications. South Asian J Cancer 2014;3(3):185. DOI: 10.4103/2278330X.136812.

6. Nam KY. The validity of oral prosthetic stents for maxillofacial cancer during radiation therapy: case report. Dent Oral Maxillofac Res 2020;6(2):1. DOI: 10.15761/DOMR.1000336.

7. Yangchen $\mathrm{K}$, Singh $\mathrm{S}$, Aggarwal $\mathrm{H}$, et al. Cerrobend shielding stents for buccal carcinoma patients. J Can Res Ther 2016;12(2):1102. DOI: 10.4103/0973-1482.144688.

8. Wilke CT, Zaid M, Chung C, et al. Design and fabrication of a 3Dprinted oral stent for head and neck radiotherapy from routine diagnostic imaging. 3D Print Med 2017;3(1):12. DOI: 10.1186/s41205017-0021-4.

9. Zaid M, Bajaj N, Burrows H, et al. Creating customized oral stents for head and neck radiotherapy using 3D scanning and printing. Radiat Oncol 2019;14(1):148. DOI: 10.1186/s13014-019-1357-2.

10. El Hawari W, Rokhssi H, Azhari M, et al. Radiotherapy and management in maxillofacial prosthodontic: part 3 - proposal for a joint sheet between the specialist in maxillofacial prosthodontics and the radiotherapist/oncologist. Integr J Med Sci 2021;8:2. DOI: 10.15342/ ijms.2021.388.

11. Bruno JS, Miranda-Silva W, da V, et al. Digital workflow for producing oral positioning radiotherapy stents for head and neck cancer. $J$ Prosthod 2020;29(5):448-452. DOI: 10.1111/jopr.13155.

12. Jain $A R$, Janani $T$. Clinical demonstration of various radiation stentsan overview. J Pharm Sci 2016;8:10.

13. Yangchen $\mathrm{K}$, Siddharth $\mathrm{R}$, Singh $\mathrm{S}$, et al. A pilot study to evaluate the efficacy of cerrobend shielding stents in preventing adverse radiotherapeutic effects in buccal carcinoma patients. J Can Res Ther 2016;12(1):314. DOI: 10.4103/0973-1482.154015.

14. Grant SR, Williamson TD, Stieb S, et al. A dosimetric comparison of oral cavity sparing in the unilateral treatment of early stage tonsil cancer: IMRT, IMPT, and tongue-deviating oral stents. Adv Radiat Oncol 2020;5(6):1359-1363. DOI: 10.1016/j.adro.2020.08.007.

15. Farajollahi AR, Bouzarjomehri F, Kiani M. Comparison between clinically used irregular fields shielded by cerrobend and standard lead blocks. J Biomed Phys Engineer 2015;5(2):77.

16. Doi H, Tanooka M, Ishida T, et al. Utility of intraoral stents in external beam radiotherapy for head and neck cancer. Rep Pract Oncol Radiother 2017;22(4):310-318. DOI: 10.1016/j.rpor.2017.03.002.

17. Chen $D$, Chen $X$, Chen $X$, et al. The efficacy of positioning stents in preventing oral complications after head and neck radiotherapy: a systematic literature review. Radiat Oncol 2020;15(1):90. DOI: 10.1186/ s13014-020-01536-0. 